\title{
Effect of antimicrobial feed additives on rabbit caecal methanogenesis in vitro
}

\author{
M Marounek 1, V Skrivanova 2, OG Savka ${ }^{1}$ \\ $1 /$ nstitute of Animal Physiology and Genetics, Czech Academy of Sciences, CZ 104 00, Prague 10 - Uhrineves ; \\ 2Reasearch Institute of Animal Production, CZ 104 O0 Prague 10 Uhrineves, Czech Republic
}

lonophore antibiotics and other antimicrobial feed additives decrease methanogenesis in the digestive tract of ruminants, primarily due to their action on $\mathrm{H}_{2}$-producing bacteria. In rabbits, various dietary factors that influence methanogenesis in the hindgut are not completely known. The purpose of this study was to specify effect of eight antimicrobial feed additives on production of methane and volatile fatty acids (VFA) in vitro incubations of rabbit ceacal content.

Ceacal content of rabbits was diluted with buffer and incubated anaerobically in hermetically closed serum bottles for $8 \mathrm{~h}$ at $38^{\circ} \mathrm{C}$ with starch, pectin and xylan. Antimicrobials were added at 0 (control) or $5 \mathrm{mg} / \mathrm{l}$. At the end of the incubation the manometric pressure in the vessels and methane content in the headspace gas were measured. VFA were estimated by titration after steam distillation, methane by GLC.
Lincomycin was the only additive that significantly decreased both methanogenesis $(-19.1 \%)$ and VFA production $(-30.4 \%)$. Tylosin lowered the VFA production $(-16.5 \%)$, but its effect on methanogenesis was non significant. Avoparcin, bacitracin and spiramycin had only marginal influence on production of methane and VFA. Surprisingly, ionophore antibiotics (maduramicin, monensin and salinomycin) stimulated rabbit ceacal methanogenesis by $15.5 \%$ on average. The effect of ionophores was statistically significant $(P<0.02)$. Ionophores, which are common coccidiostats, probably suppress some ceacal $\mathrm{H}_{2}$-utilizing bacteria and more $\mathrm{H}_{2}$ is then available for methanogens. It can be concluded that the effect of antimicrobial feed additives on methanogenesis differs in rabbits from that in ruminants. 